\title{
AfECTO POSITIVO Y NEGATIVO: ¿UNA DIMENSIÓN BIPOLAR O DOS DIMENSIONES UNIPOLARES INDEPENDIENTES?*
}

\section{Positive ANd Negative AfFect: ONe BIPOLAR DIMENSION OR TWO INDEPENDENT UNIPOLAR DIMENSIONS?}

\author{
Ferran Padrós Blázquez ${ }^{* *}$, Carles Soriano-Mas ${ }^{* *}$ y Gabriela Navarro Contreras ${ }^{* * *}$
}

\author{
*Trabajo realizado en el marco del Proyecto N 862/PROMEP/2009: Aplicación y estudio \\ psicométrico de la Escala de Gaudibilidad en población mexicana y en distintos trastornos \\ clínicos. Asimismo, este trabajo ha sido posible en parte gracias a que el Dr. Soriano-Mas es \\ beneficiario de un contrato 'Miguel Servet' del Instituto de Salud Carlos III (CP10/00604). \\ ${ }^{* *}$ Doctor en Psicología. Profesor-investigador de la Facultad de Psicología de la Universidad \\ Michoacana de San Nicolás de Hidalgo. Morelia, Mich (México). \\ E-Mail: fpadros@umich.mx; fpadros@uoc.edu \\ ***Doctor en Psicología. Investigador del Instituto de Investigación Biomédica de Bellvitge (IDIBELL). \\ Centro de Investigación Biomédica en Red de Salud Mental (CIBERSAM), Instituto de Salud \\ Carlos III. Barcelona, España. \\ ${ }^{* * * *}$ Doctora en Psicología. Profesora-investigadora de la Facultad de Psicología de la Universidad \\ Michoacana de San Nicolás de Hidalgo. Morelia, Mich (México). \\ Facultad de Psicología, Universidad Michoacana de San Nicolás de Hidalgo. \\ C/ Francisco Villa No. 450, Colonia: Doctor Miguel Silva, C.P. 58120 Morelia, Mich - México.
}

\section{RESUMEN}

Desde los trabajos pioneros de Bradburn (1969) el afecto positivo y el afecto negativo han sido concebidos como dos dimensiones unipolares independientes, aunque todavía se mantiene hoy en día la controversia teórica entre defensores de un modelo unidimensional bipolar y los de un modelo bidimensional unipolar. El objetivo de este artículo es revisar la problemática que rodea esta controversia tomando en consideración por un lado, los dos estados afectivos (el humor o tono afectivo y las emociones) y por otro lado, revisando los estudios y modelos teóricos provenientes de distintas áreas tales como Psicometría, Psicología Básica, neurociencia, Psicología Clínica y Psicología de la Salud. Finalmente, se ofrece una posible explicación a los datos contradictorios obtenidos en los distintos estudios. Se sugiere la conveniencia de hacer estudios claramente diferenciados sobre el humor y las emociones. Sobre el humor existen pocos trabajos, pero éstos ofrecen mayor apoyo a un modelo unidimensional bipolar. Sin embargo, sobre las emociones hay mayor cantidad de estudios que ofrecen mayor apoyo a un modelo bidimensional unipolar.

Palabras clave: Afecto positivo; Afecto negativo; Estado afectivo; Humor; Emociones.

\section{ABSTRACT}

Since the seminal contribution of Bradburn (1969), positive and negative affect have been 
conceived as two independent unipolar dimensions. However, the controversy between these ideas and the supporters of a single bipolar dimension is nowadays still alive. In this review we identify two problems. The first one is of a conceptual nature, because, as Russell and Carroll (1999) pointed out, the term affection is not unambiguously defined and rarely researchers in this field make an explicit definition. According to Rosenberg (1988), there are at least two types of affective states, which are widely acknowledged: emotions and mood, or affective tone. Emotions are characterized for, and differ from $\operatorname{mood}$ in, appearing in response to an event, being intense, short-lived and specific. They are also characterized by provoking important physiological reactions in the individuals, as well as recognizable patterns of facial expression, and by determining individuals to behave congruently in relation to the emotion they are experiencing. Combining these two very different components of affect hinders the comparison of the work of different authors, and also the development of theoretical models combining the different aspects included within this concept.

The second problem is related to the measurements used in the research assessing affect, where the possible existence of events or circumstances causing the affective state is not normally considered. Likewise, measurement regarding the duration of the affect are rarely performed, and objective measurements such as physiological correlates or facial expressions are not frequently collected. Consequently, instruments do not normally distinguish between mood and emotion.

The aim of this paper is to review the most relevant questions regarding this debate taking into account, on the one hand, the two affective states included in the concept of affectivity (i.e., mood and emotion) and, on the other hand, the empirical and theoretical contributions provided by a wide range of disciplines such as Psychometry, Basic Psychology, neuroscience, and Clinical and Health Psychology. From Basic Psychology and Psychometry we reviewed the three fundamental argumentations advocating for an independence of positive and negative affect, as identified by Diener and Emmons (1984) and Russell and Carroll (1999): (1) that the correlation between items that evaluate the positive affect and negative affect is low, (2) that the correlation of items within the categories of positive affect and negative affect is high, and (3) that the two dimensions of affect correlate differently with other variables. A fourth argument is discussed referring to the variations of both affect types observed throughout a lifetime (Bushman \& Crowley, 2010; Windsor \& Anstey, 2010). From the neuro-scientific perspective we reviewed studies providing data on brain structures and neurotransmitters involved in both affect types, noting that all these studies have been carried out by inducing intense moods, which indeed might be classified as emotions rather than humor. From Psychopathology and Clinical Psychology we reviewed those disorders in which states of positive and negative mood can be experienced in a very intensive manner (positive and negative emotions), in very short periods of time and even at the same time. We also reviewed research studying the independent presence of positive and negative affect in several disorders, as well as some theoretical models on depression that indicate the possibility and desirability of adopting a 'dual' perspective in the assessment and the intervention strategies of both types of emotions in the depressive disorder and other disorders.

A proposal about the reason of such contradictory findings is presented, and we suggest that research about this topic should be better conducted setting apart mood from emotions using tools that allow measuring those elements capable of distinguishing between affect types. Although the few existing studies about affective traits and mood seem to support the bipolar one-dimensional model, the conclusions drawn from the numerous investigations about emotions favor the two independent unipolar dimensions model.

Key words: Positive affect; Negative affect; Affective state; Mood; Emotion.

\section{INTRODUCCIÓN}

A partir de los trabajos pioneros de Bradburn (1969) el afecto positivo y el afecto negativo fueron descriptos como dos 
dimensiones unipolares independientes. Concibiendo así al afecto positivo como un constructo dimensional (no categorial) que incluye todos aquellos estados afectivos con valencia positiva como alegría, entusiasmo, enamoramiento, etc., los cuales son independientes del constructo afecto negativo que también es concebido como dimensional e incluye los estados afectivos negativos tales como tristeza, ira, miedo, ansiedad, etc. Es decir, desde este modelo podrían reconocerse personas que frecuentemente experimentan estados afectivos positivos y rara vez, negativos (las personas típicamente felices), o a la inversa (personas infelices). Pero también predice la existencia de personas que experimentan bajos niveles de ambos tipos de afecto (personas con afecto aplanado) así como la existencia de personas que experimentan frecuentemente niveles elevados de afecto positivo y negativo. Según señalan Russell y Carroll (1999), pronto surgieron autores que defendían el modelo bipolar unidimensional, que concibe el afecto como una única dimensión donde puede ubicarse el estado afectivo que las personas experimentan y éste oscila entre el afecto positivo máximo y el afecto negativo máximo. De modo que cuanto más cercano está al polo positivo, más alejado lo está del negativo y viceversa. Todavía se mantiene la controversia teórica entre defensores de ambos modelos, el unidimensional bipolar y el bidimensional unipolar.

En este artículo la propuesta es revisar la problemática que rodea esta controversia y ofrecer una posible explicación a los datos contradictorios obtenidos en los distintos estudios. El primer problema es conceptual. Russell y Carroll (1999) señalan que el término afecto no está definido en consenso y rara vez los autores que investigan en este campo, explicitan una definición.

Rosenberg (1998) afirma que el afecto es un término que se refiere a todo aquello emocional, es decir, pueden ser varias formas específicas de afecto como los sentimientos, preferencias, emociones, humor y rasgos afectivos. Siguiendo a Rosenberg (1998) existen como mínimo dos clases de estados afectivos ampliamente reconocidos: las emociones y el humor o tono afectivo, que estarían incluidos en el constructo de afecto. La emoción se caracteriza por ser consecuencia de un evento, por ser intensa, poco duradera y específica, también por generar en el individuo importantes alteraciones fisiológicas, de expresión facial y predispone a éste a comportarse de manera congruente con ella. El humor, en cambio, ocupa un espacio intermedio entre la emoción y el rasgo afectivo y por lo tanto comparte características de ambos. El humor al igual que las emociones es un estado afectivo fluctuante, pero se distingue de ellas por ser un estado afectivo no relacionado con un estímulo identificable, menos intenso, más global, difuso y duradero. Además, el humor no produce importantes alteraciones en el organismo como en el caso de las emociones, pero predispone al individuo a tener respuestas emocionales congruentes con el humor o tono afectivo. La inclusión de dos componentes distintos del afecto dificulta la comparación de los trabajos de diferentes autores, así como la generación de modelos que aúnen los diferentes aspectos que se incluyen bajo este concepto.

El segundo problema son las mediciones utilizadas en las investigaciones que evalúan el afecto. En la mayoría de las investigaciones, cuando se evalúan los estados afectivos no se controla si hubo un evento o circunstancia que haya provocado el estado afectivo, tampoco suelen medir la duración del mismo, ni suelen recoger mediciones objetivas como correlatos físiológicos o expresiones faciales. De modo que los instrumentos no permiten distinguir si lo evaluado es humor o emoción. Así pues, cuando se evalúa el afecto negativo se recoge información tanto de emociones (tristeza, miedo, ira, etc.) como de humor negativo. Del mismo modo, cuando se evalúa el afecto positivo se mide tanto la presencia o ausencia de emociones (entusiasmo, alegría, disfrute, etc.) como del humor positivo.

Finalmente, debe señalarse que la mayoría de los trabajos e investigaciones sobre el 
afecto lo hacen desde una o dos perspectivas y rara vez aluden a otras disciplinas.

En el estudio que se informa se hace una revisión considerando por un lado, los dos estados afectivos (el humor y las emociones) por separado, y por el otro, el apoyo teórico y empírico del modelo bidimensional unipolar versus el modelo unidimensional bipolar del afecto positivo y negativo, teniendo en cuenta las aportaciones realizadas desde la Psicometría, la Psicología Básica, los estudios de neurociencia, la Psicología Clínica y la Psicología de la Salud.

\section{Aportaciones de la Psicometría y de la PsI- COLOGÍA BÁSICA}

Los argumentos de los defensores de la independencia del afecto positivo y el afecto negativo como señalan Diener y Emmons (1984) y Russell y Carroll (1999) están basados en: (1) que la correlación entre los ítemes que evalúa el afecto positivo y el afecto negativo es baja, (2) la correlación de los ítemes dentro de las categorías de afecto positivo y de afecto negativo es alta y (3) las dos dimensiones de afecto correlacionan de forma diferente con otras variables. Y podría incluirse un cuarto argumento referido a las variaciones de ambos tipos de afecto observados a lo largo de la vida (Bushman \& Crowley, 2010; Windsor \& Anstey, 2010).

Russell y Carroll (1999) ponen en duda la demostración de dicha independencia, argumentando:

a.- que no se han tenido en cuenta los sesgos sistemáticos de medida,

b.- el amplio rango de correlaciones entre ambos afectos halladas en distintos estudios,

c.- la naturaleza multidimensional del afecto (pero desde el modelo circumplejo defendido por Russell, 1980),

d.- la relevancia del factor tiempo como variable que modifica enormemente las correlaciones entre el bienestar y el malestar, y

e.- el efecto compensatorio producido por la integración de dos tipos de correlaciones; por un lado, las de la intensidad del afecto positivo y del negativo, las cuales son altas y positivas, y por otro lado, las de la frecuencia entre ambos tipos de afecto que son altas y negativas (Diener, Larsen, Levine \& Emmons, 1985).

Todo ello repercute en los cuatro argumentos sobre los que se fundamenta la defensa de la independencia de los afectos positivo y negativo.

\section{Sesgos SISTEMÁtICOS DE MEDIDA}

Russell y Carroll (1999) advierten acerca del efecto que tienen los sesgos conocidos (como por ejemplo el de aquiescencia que se refiere a la tendencia por parte del evaluado a contestar afirmativamente a los reactivos de los cuestionarios) en la recolección de datos y en su posterior análisis estadístico, y además, subrayan la relevancia que tienen las consideraciones semánticas de los instrumentos de medida que habitualmente son olvidadas por los investigadores de este ámbito.

Russell y Carroll (1999) señalan que los sesgos sistemáticos de respuesta son inducidos directamente por el tipo de pregunta que se realiza, de modo que entre los instrumentos utilizados pueden distinguirse distintos tipos: desde los que son estrictamente unipolares hasta los estrictamente bipolares, pasando por otros que podrían catalogarse de ambiguos. Los primeros preguntan sobre la presencia o ausencia de un afecto (ej.: si te sientes feliz: marca sí; en tal caso, por favor indica en qué grado: 1 = ligeramente hasta 7 = extremadamente). Los estrictamente bipolares formulan la cuestión dando opciones antagónicas (ej.: indica con un círculo el número que describe tu humor ahora: $7=$ muy triste, $0=$ neutral, $7=$ muy feliz). Y los ambiguos están entre ambos 
extremos, sin dar pistas claras sobre el modelo a tener en cuenta en el momento de responder (ej.: pon un círculo en el número que describa el grado en el cual te sientes feliz: 1 = definitivamente no me siento, $2=$ no puedo decidirme, 3 = ligeramente me siento, y 4 = definitivamente me siento). Russell y Carroll (1999) sostienen que el formato del instrumento de medida condiciona claramente las posteriores correlaciones y explica gran parte de las grandes diferencias halladas en las correlaciones entre ambos afectos que se observan en distintos estudios. Debe considerarse que existen estudios realizados por defensores de la bipolaridad (Diener \& Emmons, 1984; Diener et al., 1985), que utilizan instrumentos unipolares y bipolares, pero no estudian las posibles diferencias atribuibles al tipo de instrumento utilizado, ni tampoco muestran los resultados de forma separada en función del tipo de medición usada.

\section{EL AMPLIO RANGO DE CORRELACIONES ENTRE AMBOS AFECTOS}

Por otro lado, Russell y Carroll (1999) subrayan las grandes diferencias halladas entre los diversos estudios que relacionan el afecto positivo con el afecto negativo. Las mismas oscilan entre -.08 (Sandín et al., 1999) y -.92 (este último obtenido por Russell y Carroll, 1999, controlando los errores de medición).

\section{LA NATURALEZA MULTIDIMENSIONAL DEL AFECTO} DESDE EL MODELO CIRCUMPLEJO

Russell (1980) defiende un modelo circumplejo del afecto, el cual puede definirse a través de dos ejes. Uno es el descripto por feliz - infeliz y el otro por arousal - dormido (activado - desactivado), y todos los afectos que pueden ser descriptos se ubicarían en algún grado de la circunferencia que puede dibujarse a partir de los dos ejes. Russell y Carroll (1999) también señalan que algunas escalas, entre ellas la Escala PANAS (Posi- tive Affect and Negative Affect Scales) de Watson, Clark y Tellegen (1988), que ha recibido reciente apoyo confirmatorio con respecto a su estructura bifactorial (Tuccitto, Giacobbi \& Leite, 2010) y las Escalas de Balance Afectivo (EBA) de Warr, Barter y Brownbridge (1983) son escalas constituidas por ítemes en los que no están bien representados los diferentes matices y tampoco aparecen los valores extremos de una misma dimensión, es decir que en la mayoría de los casos los adjetivos que componen una escala no tienen el antónimo en la otra (como por ejemplo, tenso - relajado o eufórico - deprimido). Según Russell y Carroll (1999) tanto las escalas de afecto positivo como las de afecto negativo están constituidas por ítemes que miden en la mayoría de los casos, estados afectivos que se ubican en la zona arousal elevado o medio, de modo que las escalas de afecto positivo evalúan en realidad activación o arousal junto afecto positivo y las escalas de afecto negativo miden arousal más afecto negativo. En el modelo circumplejo de Russell (1980) estarían separados por $90^{\circ}$ aproximadamente (y no por $180^{\circ}$ ), lo cual según Russell y Carroll (1999) explicaría las bajas correlaciones halladas entre las escalas de afecto positivo y negativo de la PANAS. Sin embargo, en estudios como los de Diener y Emmons (1984, estudio 3) se ha controlado el afecto de arousal y se obtuvieron correlaciones de .26 entre-sujetos y de -.54 intra-sujetos entre los dos tipos de afecto.

\section{LA RELEVANCIA DEL FACTOR TIEMPO}

Según observaron Russell y Carroll (1999) y Diener (1984), a medida que se realizan evaluaciones del afecto en períodos más cercanos, la independencia de ambos tipos de afecto decrece. Como señalan Diener y Emmons (1984), es altamente improbable que una persona pueda experimentar o sentir ambos afectos en niveles altos en el mismo momento, debido a que han detectado que cuando se siente fuertemente un tipo de afecto, éste tiende a suprimir al otro 
tipo durante períodos cortos de tiempo. Así, se observa que si se evalúan períodos de tiempo de una semana o más la independencia se mantiene, pero cuando se trata de períodos de tiempo más cortos, ésta desaparece.

Pareciera que pueden identificarse momentos en los cuales las personas pueden experimentar los dos tipos de afecto con alta intensidad a la vez. En el trabajo de Larsen, McGraw y Cacioppo (2001) se observa después de la proyección de una película ( $\mathrm{La}$ vida es bella), cómo algunos sujetos (el 44\%) manifiestan en el mismo momento altos niveles de tristeza y de felicidad (entendida como emoción). También puede observarse que muchas personas experimentan niveles elevados de afecto positivo y negativo en parques de atracciones o practicando deportes de aventura.

\section{EL EFECTO COMPENSATORIO PRODUCIDO POR LA INTENSIDAD Y LA FRECUENCIA}

Diener (1984), Diener y Emmons (1984) y Russell y Carroll (1999) explican que si se controla la frecuencia del afecto positivo y negativo la correlación es positiva. Es decir, que los sujetos que manifiestan haber experimentado un malestar más intenso también informan un mayor bienestar, y los sujetos que informan experimentar bajos niveles de bienestar también reportan bajos niveles de malestar.

Merece especial atención el estudio llevado a cabo por Diener y colaboradores (1985), en el cual se controló el efecto de los sesgos clásicos de respuesta, es decir, los de respuesta con tendencia central por parte de algunas personas y tendencia a los extremos por parte de otras. En el citado estudio, a priori, observaron una correlación positiva y significativa entre la intensidad de afecto positivo y negativo en valores alrededor del .70. Luego, controlando los efectos de la intensidad, se observa que la correlación entre los valores medios de la afectividad positiva y negativa oscila entre -.46 y -.86 . El valor menor sugiere que el afecto positivo es un constructo diferente al afecto negativo pero relacionado a él; sin embargo el valor mayor indica que ambos afectos son los polos opuestos de una misma dimensión.

Por otro lado, en el trabajo de Diener, Smith y Fujita (1995) realizado utilizando exclusivamente emociones (lo cual puede ser interpretado como el estudio del afecto de alta intensidad) se ha extraído un factor de afecto positivo y otro de afecto negativo, los cuales a su vez están integrados respectivamente, por dos tipos de emociones positivas (alegría y amor) y por cuatro tipos de emociones negativas (miedo, tristeza, ira y vergüenza).

En cuanto a la frecuencia, en distintos estudios (Diener et al., 1985; Kardum, 1999; Russell \& Carroll, 1999) se ha observado que correlaciona de forma significativa y negativa. En otras palabras, las personas que informan sentir con alta frecuencia afectos positivos refieren experimentar raramente afectos negativos y viceversa. Esto, junto con las altas correlaciones positivas en la intensidad de ambos afectos (observadas en trabajos donde no se distingue entre tono afectivo y emociones), permite pensar que las bajas correlaciones halladas entre el afecto positivo y negativo en trabajos como los de Watson, Clark y Carey (1988) y Sandín y colaboradores (1999) son debido al efecto compensatorio de ambas correlaciones (Diener et al., 1985; Russell \& Carroll, 1999).

En el trabajo de Diener y Emmons (1984) que recoge y contabiliza por separado el afecto positivo en media o alta intensidad y hace lo mismo con el afecto negativo (lo que podría entenderse como una medición de las emociones) se observa que la correlación tiende a desaparecer (a medida que se utilizan períodos de tiempo más extensos) lo cual da apoyo al modelo bidimensional unipolar. Pero al referimos a la información relativa al humor o tono afectivo, éstas tienden a ser negativas, muy elevadas y significativas, lo cual sugiere un modelo unidimensional bipolar. De esta manera, se hace especialmente relevante y necesario distinguir entre las emociones y el humor. 
Existe una larga tradición en el estudio de la diferenciación y clasificación de las emociones (Ekman, Friesen \& Ellsworth, 1972; Izard, 1978) en la que se distinguen entre positivas y negativas ( $\mathrm{y}$ en algún caso también se añade alguna emoción neutra, como la sorpresa). Por otro lado, un importante argumento a favor de la independencia de las emociones positivas y negativas es la ley de la asimetría hedónica descripta por Frijda (1988), la cual postula que mientras las emociones placenteras tienden a diluirse aunque se mantenga la situación que las provoca, las emociones dolorosas tienden a persistir si también lo hacen las condiciones que las han provocado. Por otro lado, Fredrickson (1998) sugiere que las emociones positivas pueden diferenciarse substancialmente de las negativas, en que las emociones positivas tienen un efecto expansivo en la conciencia (y favorecen procesos tales como la creatividad), mientras las negativas tienden al estrechamiento de la misma (reduciendo la flexibilidad mental).

\section{LA PROPUESTA DE UN MODELO INTEGRADOR}

En la revisión llevada a cabo por Reich, Zautra y Davis (2003) se propone el modelo dinámico del afecto (The Dynamic Model of Affect - DMA) para explicar los datos contradictorios aportados por los defensores del modelo unidimensional bipolar y del modelo bidimensional unipolar. El modelo predice que bajo situaciones de elevado estrés, tienden a aparecer el afecto positivo y el afecto negativo como una sola dimensión bipolar, es decir, relacionados de forma inversa. En cambio, en situaciones de bajo nivel de estrés ambos tipos de afecto tienden a la ausencia de relación, manifestándose como el modelo bidimensional unipolar propone. El modelo especifica que bajo condiciones de estrés la capacidad de procesamiento del organismo se reduce, simplificando y acelerando el proceso de decisión y actuación. Las ventajas adaptativas del procesamiento simple justificaría el funcionamiento congruente con el modelo unidi- mensional. En cambio, en situaciones de baja presión de afrontamiento y especialmente en situaciones de buen funcionamiento del individuo el uso de un procesamiento más complejo que permita la valoración de ambos tipo de afecto de forma separada e independiente favorece una mejor adaptación. El modelo cuenta ya con apoyo empírico (Zautra, Potter \& Reich, 1997; Zautra, Reich, Davis, Nicolson \& Potter, 2000).

Aunque debe tenerse presente que los datos aportados sobre el modelo DMA son contradictorios con algunos aportados desde el ámbito clínico por el modelo tripartito (Clark \& Watson, 1991; Joiner, Catanzaro \& Laurent, 1996; Watson et al., 1995), el cual se caracteriza por distinguir tres tipos de afecto independientes: el afecto negativo, el afecto positivo y el nivel de arousal. En un estudio, Watson y colaboradores (1988) observaron que los pacientes con trastornos de ansiedad (es decir, con niveles elevados de estrés) correlacionaban significativamente con elevados niveles de emociones negativas; en cambio no hallaron correlaciones significativas con las emociones positivas (excepto en la fobia social).

\section{DATOS APORTADOS DESDE LA NEUROCIENCIA}

Davidson (1983, 1992; Davidson, Ekman, Saron, Senulis \& Friesen, 1990) señaló la activación de determinadas y distintas zonas cerebrales en cada tipo de afecto. Posteriormente, en una revisión de la incidencia selectiva de los mecanismos neurológicos (corticales y subcorticales) que subyacen al sistema afectivo, se defiende la existencia de un doble sistema afectivo (uno positivo y otro negativo) que actúa en paralelo y que luego se integra dando lugar a un comportamiento de aproximación (si resulta más grato), neutro o de retirada (si resulta más aversivo) (Cacioppo, Berntson, Larsen, Poehlmann \& Ito, 2000; Cacioppo, Gardner \& Berntson, 1999). Estos mismos autores también han señalado que una alta activación en el afecto positivo y en el negativo en el mismo momento provoca que el individuo experimente ambivalencia, 
y en el caso de que ambos afectos se experimenten en un bajo nivel entonces da lugar a indiferencia. Estudios recientes han acentuado el rol de vía dopaminérgica mesolímbica en el refuerzo y la relevancia de la amígdala en la aversión. También son destacables los aportes obtenidos a partir de los estudios neuroanatómicos en los que Lane y colaboradores (1997) mediante técnicas de neuroimagen, observaron que la felicidad (entendida como afecto positivo) se distingue de la tristeza en que se advierte una mayor actividad en la zona medial ventral del córtex frontal, y que la tristeza está relacionada con un aumento de la activación en la ínsula anterior.

Por otro lado, se han reportado diferencias en la participación de neurotransmisores en ambos tipos de afecto. Se ha observado que la administración de recaptadores de la serotonina reduce el afecto negativo, pero no aumenta el afecto positivo (Knutson et al., 1998) y que la actividad dopaminérgica aumenta el afecto positivo y no se halla relacionada con el afecto negativo (Caccioppo et al., 1999).

Debe notarse que todos estos estudios han sido realizados induciendo estados de ánimo intensos, que pueden ser catalogados como emociones y no como humor (véase anteriormente las diferencias entre humor y emoción).

\section{Aportaciones de la Psicología Clínica y de la Psicología de La SALUd}

\section{EL ESPECTRO DE LA BIPOLARIDAD}

Desde el ámbito de la Psicopatología, se describieron alteraciones y trastornos que dan apoyo a la idea de que estados de ánimo positivos y negativos pueden ser experimentados de forma muy intensa (emociones positivas y negativas) en períodos de tiempo muy breves e incluso en el mismo momento. Así pues, dentro de los trastornos del estado de ánimo, es destacable el episodio mixto, en el cual los sujetos que lo padecen experimentan estados de ánimo que se al- ternan con rapidez (tales como tristeza, irritabilidad, euforia, etc.; nótese que son nombres de emociones), acompañados de otros síntomas del episodio maníaco y del depresivo mayor (American Psychiatric Association, 2000). Por otro lado, también se destaca el fenómeno de labilidad emocional, que se refiere a cambios abruptos afectivos, en los cuales el paciente se caracteriza por estar contento y alegre al tratar un tema y llorar súbitamente al cambiar a otro (Va1lejo, 2006).

Deben considerarse todos estos casos, y otros que también producen efectos similares (los producidos por enfermedades médicas o por la ingestión de sustancias), como claros ejemplos de que las personas pueden experimentar ambos tipos de emociones en períodos de tiempo muy cortos o incluso, en el mismo momento.

\section{HALLAZGOS EN LOS TRASTORNOS DE PERSONALIDAD}

Debe destacarse también en el ámbito clínico, la descripción que realizan Millon y Davis (1998) sobre los trastornos de la personalidad, caracterizando tres tipos: el esquizoide, el evitativo y el depresivo. En los tres tipos, las personas que los padecen manifiestan una gran dificultad para experimentar placer y experiencias alegres y positivas de la vida y además estos autores señalan que es debido a causas diversas.

En el caso del trastorno de personalidad esquizoide, es atribuido a una incapacidad intrínseca del individuo. Asimismo, estas personas se caracterizan por manifestar bajos niveles de afecto negativo, justamente lo contrario a lo que ocurre en los otros dos trastornos de personalidad.

El trastorno de personalidad por evitación se daría como consecuencia de una preocupación excesiva y una hipersensibilidad a las tensiones, lo cual supone una gran inversión de sus energías, perdiendo las necesarias para atender y buscar situaciones placenteras y agradables.

Finalmente, el trastorno de personalidad depresiva induce a las personas a no esperar 
la experimentación de recompensas, placeres y experiencias positivas de la vida, lo cual también influye en gran medida en el poco placer que ellas experimentan.

Por otro lado, el modelo tripartito (Clark \& Watson, 1991) comentado anteriormente, además de haber recibido apoyo empírico, resulta especialmente útil en al ámbito de la clínica, ya que permite caracterizar y discriminar a los pacientes depresivos y los pacientes ansiosos a partir de los tres niveles de afecto. Se ha observado que las personas que padecen depresión se caracterizan por elevados niveles de afecto negativo y bajos niveles de afecto positivo (lo cual podría interpretarse, distinguiendo las emociones del humor, que los pacientes depresivos experimentan una alta frecuencia de emociones negativas, escasa frecuencia de emociones positivas y un humor negativo). Las personas que padecen trastornos de ansiedad se caracterizan por elevados niveles de afecto negativo y elevados niveles de arousal (lo cual puede ser interpretado como una manifestación frecuente de emociones negativas, de altos niveles de arousal y una frecuencia de emociones positivas similar a la población general).

Goodhart (1985) observó que el pensamiento positivo en situaciones de afrontamiento a situaciones estresantes no reducía los niveles de afecto negativo. Existen otros estudios en esta dirección (Felton \& Revenson, 1984; Zautra et al., 1995) que llevan a Reich y colaboradores (2003) a señalar que

"la modificación de cogniciones desadaptadas (pensamiento negativo e irracional) parece ser altamente efectiva en la reducción del estrés, pero puede que no ayude a la promoción de la afectividad positiva. Además, promocionar pensamientos positivos puede no reducir el afecto negativo" ( p. 77).

\section{ESTUDIOS SOBRE LA DEPRESIÓN}

En el estudio teórico de la depresión han surgido modelos que enfatizan el papel de los acontecimientos que producen emociones negativas y favorecen un humor negativo, como causantes y mantenedores del trastorno depresivo, como por ejemplo la desesperanza aprendida de Seligman (1975) o los modelos cognitivos de Beck (Beck, Rush, Shaw \& Emery, 1998) y de Ellis (Ellis \& Grieger, 1981). Se ha estudiado preferentemente el efecto de variables con connotación negativa tal como la rumiación negativa (NolenHoeksema, 2000) en la depresión. Como se ha señalado (Wichers, Jacobs, Derom, Thiery $\&$ van Os, 2008), a pesar de que el afecto negativo en la depresión ha sido ampliamente estudiado, el afecto positivo recibió mucha menos atención. Desde 1973 existen modelos que subrayan el papel de los acontecimientos susceptibles de producir emociones positivas (habitualmente se refieren al disfrute) como elemento crucial en la depresión. Ya desde las teorías conductuales se indicaba que los sujetos deprimidos se caracterizan por estar sometidos a una baja tasa de refuerzos (Lewinsohn \& Amenson, 1978; Lewinshon \& Graf, 1973). Posteriormente, Davis y Burns (1999) advierten que la depresión también puede aparecer cuando una persona falla en la habilidad para producir emociones positivas ante un mínimo número de comportamientos y estímulos ambientales, y que en dicha circunstancia la frecuencia de eventos positivos es irrelevante.

Debe comentarse que el estudio por separado del afecto positivo y negativo se ha llevado a cabo y parece resultar de utilidad en otros trastornos mentales tales como la esquizofrenia (Blanchard, Mueser \& Bellack 1998; Horan, Wynn, Kring, Simons \& Green, 2010), el trastorno bipolar (Meyer \& Baur, 2009) y trastornos relacionados con sustancias (Van Etten, Higgins, Budney \& Badger, 1998). También se ha estudiado en trastornos físicos como el cáncer (Voogt et al., 2005), cardiopatías (Spindler, Denollet, Kruse \& Pedersen, 2009; Versteeg et al., 2009) y otros trastornos de salud (Fortenberry et al., 2009; Janicki-Deverts, Cohen, Doyle, Turner \& Treanor, 2007).

Teniendo en cuenta las diferentes explicaciones psicológicas referidas a la depre- 
sión, trastornos de personalidad y otros que no han sido considerados, que aluden a alteraciones en la frecuencia de los dos tipos de emociones, resulta lógico plantearse la posibilidad de adoptar una perspectiva dual ante el trastorno depresivo y otros (la perspectiva es triple si se añade el arousal como sugiere el modelo tripartito) y estudiar de forma separada, los moduladores que regulan las emociones negativas y los que regulan las emociones positivas. Ello implicaría a su vez, una evaluación e intervención para ambos tipos de emociones en el ámbito aplicado dando lugar a tratamientos coadyuvantes como los aplicados en el tratamiento de la depresión (Fava, Rafanelli, Cazzaro, Conti \& Grandi, 1998), así como estudiar los efectos de los tratamientos en distintos trastornos en ambos tipos de afecto (Dichter, Tomarken, Freid, Addington \& Shelton, 2005; Strong et al., 2009).

\section{Discusión}

En este trabajo se ha realizado una revisión sobre el debate referido a la concepción del afecto positivo y el afecto negativo. Se consultaron los aportes realizados desde la Psicometría, la Psicología Básica, los estudios de neurociencia, la Psicología Clínica y la Psicología de la Salud.

Existen dos posturas contrapuestas: los defensores de un modelo unidimensional bipolar y los que apoyan un modelo bidimensional unipolar. Se ha destacado que el concepto de afecto incluye dos estados afectivos (emociones y humor o tono afectivo) que tienen características muy diferentes y que en las mediciones no se suelen distinguir. Por ello se sugiere la conveniencia de hacer estudios claramente diferenciados sobre las emociones y el humor afectivo y utilizando instrumentos que permitan controlar aquellos elementos que distinguen ambos tipos de afectos, como la presencia de un evento o circunstancia que ha provocado el estado afectivo, mediciones sobre la intensidad y duración del mismo, y el uso de mediciones objetivas como correlatos fisiológicos o expresiones faciales.

Sobre el humor o tono afectivo no se conoce trabajo alguno que estudie específicamente dicho tipo de afecto (controlando no incluir emociones). Los datos que se desprenden de estudios psicométricos y de la Psicología Básica ofrecen mayor apoyo a un modelo unidimensional bipolar, pero se requerirían estudios diseñados ex profeso que corroboren la adecuación de dicho modelo.

Sobre las emociones hay mayor cantidad de estudios y en distintas disciplinas (Psicometría, Psicología Básica, neurociencia, Psicología Clínica y Psicología de la Salud). Se observa que las emociones positivas y negativas manifiestan una cierta relación, pero los datos ofrecen claramente mayor apoyo al modelo bidimensional unipolar.

Finalmente, modelos más complejos como el modelo dinámico del afecto (Reich et al., 2003) pueden aclarar algunos datos contradictorios y ser más pertinentes para integrar los datos que se derivan de distintas áreas.

\section{REFERENCIAS BIBLIOGRÁFICAS}

American Psychiatric Association. (2000). Diagnostic and statistical manual for mental disorders (4a. ed.). Washington, DC: Author.

Beck, A., Rush, A.J., Shaw, B.F., \& Emery, G. (1998). Terapia cognitiva de la depresión [Cognitive therapy of depression] ( $5^{\mathrm{a}}$ ed.). Bilbao: Desclée de Brouwer.

Blanchard, J.J., Mueser, K.T., \& Bellack, A.S. (1998). Anhedonia, positive and negative affect, and social functioning in schizophrenia. Schizophrenia Bulletin, 24(3), 413-424. doi: 10.1093/oxfordjournals.schbul.a033336.

Bradburn, N.M. (1969). The structure of psychological well-being. Chicago: Aldine. 
Bushman, B.B. \& Crowley, S.L. (2010). Is the structure of affect similar for younger and older children? Cross-sectional differences in negative and positive affectivity. Journal of Psychoeducational Assessment, 28(1), 31-39. doi: 10.1177/0734282909337584.

Cacioppo, J.T., Gardner, W.L. \& Berntson, G.G. (1999). The affect system has parallel and integrative processing components: form follows function. Journal of Personality and Social Psychology, 76(5), 839-855. doi: 10. 1037/0022-3514.76.5.839.

Cacioppo, J.T., Berntson, G.G., Larsen, J.T., Poehlmann, K.M. \& Ito, T.A. (2000). The psychophysiology of emotion. En M. Lewis \& J.M. Haviland-Jones (Eds.), Handbook of emotions (pp. 173-191). New York: Guilford.

Clark, L.A. \& Watson, D. (1991). Tripartite model of anxiety and depression: Psychometric evidence and taxonomic implications. Journal of Abnormal Psychology, 3, 316-336. doi: 10.1037/0021-843X.100.3.316.

Davidson, R.J. (1983). Affect, repression, and cerebral asymmetry. En L. Temoshok, C. Van Dyke, \& L.S. Zegans (Eds), Emotions in health and illness: Theoretical and research foundations (pp. 123-135). New York: Grune \& Stratton.

Davidson, R.J. (1992). Emotion and affective style: Hemispheric substrates. Psychological Science, 6, 239-253. doi: 10.1111/j.1467-92 80.1992.tb00254.x.

Davidson, R.J., Ekman, P., Saron, C.D., Senulis, J.A. \& Friesen, W.V. (1990). Approach-withdrawal and cerebral asymmetry: Emotionional expression and brain physiology I. Journal of Personality and Social Psychology, 58, 330-341. doi: 10.1037/002235 14.58.2.330.

Davis, P.A. \& Burns, G.L. (1999). Influence of emotional intensity and frequency of positive and negative events on depression. European Journal of Psychological Assessment, 15(2), 106-116. doi: 10.1027//1015-5759.15.2.106.
Dichter, G.S., Tomarken, A.J., Freid, C.M., Addington, S. \& Shelton, R.C. (2005). Do venlafaxine XR and paroxetine equally influence negative and positive affect? Journal of Affective Disorders, 85(3), 333-339. doi:10.10 16/j.jad.2004.10.007.

Diener, E. (1984). Subjective well-being. Psychological Bulletin, 95(3), 542-575. doi: 10.1 037/0033-2909.95.3.542.

Diener, E. \& Emmons, R.A. (1984). The independence of positive and negative affect. Journal of Personality and Social Psychology, 47(5), 1105-1117. doi: 10.1037/00223514. 47. 5.1105 .

Diener, E., Larsen, R.J., Levine, S. \& Emmons, R.A. (1985). Intensity and frequency: Dimensions underlying positive and negative affect. Journal of Personality and Social Psychology, 48(5), 1253-1265. doi: 10.1037/0022-3514. 48.5.1253.

Diener, E., Smith, H. \& Fujita, F. (1995). The personality struture of affect. Journal of Personality and Social Psychology, 69(1), 130141. doi: 10.1037/0022-3514.69.1.130.

Ekman, P., Friesen, W.V. \& Ellsworth, P. (1972). Emotion in the human face. New York: Pergamon Press.

Ellis, A. \& Grieger, R. (1981). Manual de terapia racional emotiva [Manual of rational emotive therapy]. Bilbao: Desclée de Brouwer.

Fava, G.A., Rafanelli, C., Cazzaro, M., Conti, S. \& Grandi, S. (1998). Well-being therapy: A novel psychotherapeutic approach for residual symptoms of affective disorders. Psychological Medicine: A Journal of Research in Psychiatry and the Allied Sciences, 28(2), 475-480. doi: 10.1017/S003329179 7006363.

Felton, B.J. \& Revenson, T.A. (1984). Coping with chronic illness: A study of illness controllability and the influence of coping strategies on psychological adjustment. Journal of Consulting and Clinical Psychology, 52, 343353. doi:10.1037/0022-006X.52.3.343. 
Fortenberry, K.T., Butler, J.M., Butner, J., Berg, C.A., Upchurch, R. \& Wiebe, D.J (2009). Perceived diabetes task competence mediates the relationship of both negative and positive affect with blood glucose in adolescents with type 1 diabetes. Annals of Behavioral Medicine, 37(1), 1-9. doi: 10.1007/s12160-0099086-7.

Fredrickson, B.L. (1998). What good are positive emotions? Review of General Psychology, 2, 300-319. doi: 10.1037/1089-2680.2.3.300

Frijda, N.H. (1988). The laws of emotion. American Psychologist, 43(5), 349-358. doi: 10. 1037/0003-066X.43.5.349.

Goodhart, D.E. (1985). Some psychological effects associated with positive and negative thinking about stressful event outcomes: Was Pollyanna right? Journal of Personality and Social Psychology, 48, 216-232. doi: 10.10 37/0022-3514.48.1.216.

Horan, W.P., Wynn, J.K., Kring, A.M., Simons, R.F. \& Green, M.F. (2010). Electrophysiological correlates of emotional responding in schizophrenia. Journal of Abnormal Psychology, 119(1), 18-30. doi: 10.1037/a00175 10.

Izard, C.E. (1978). Human emotion. New York: Plenum.

Janicki-Deverts, D., Cohen, S., Doyle, W.J., Turner, R.B. \& Treanor, J.J. (2007). Infectioninduced proinflammatory cytokines are associated with decreases in positive affect, but not increases in negative affect. Brain, Behavior, and Immunity, 21(3), 301-307. doi: 10.1016/j.bbi.2006.09.002.

Joiner, Jr. T.E., Catanzaro, S.J. \& Laurent, J.L (1996). The tripartite structure of positive and negative affect, depression, and anxiety in child and adolescent psychiatric inpatients. Journal of Abnormal Psychology, 105, 401409. doi: 10.1037/0021-843X.105.3.401.

Kardum, I. (1999). Affect intensity and frequency: Their relation to mean level and variability of positive and negative affect and
Eysenck's personality traits. Personality and Individual Differences, 26, 33-47. doi: 10.10 16/S0191-8869(98)00157-3.

Knutson, B., Wolkowitz, O.M., Cole, S.W., Chan, T., Moore, E.A., Johnson, R.C., Terpstra, J., Turner, R.S. \& Reus, V.I. (1998). Selection alteration of personality and social behavior by serotonergic intervention. American Journal of Psychiatry, 155, 373-379.

Lane, R.D., Reiman, E.M., Geoffrey, M.D., Ahern, L., Schwart, G.E. \& Davidson, R.J. (1997). Neuroanatomical correlates of happiness, sadness, and disgust. American Journal of Psychiatry, 154(7), 926-933.

Larsen, J.T., McGraw, A.P. \& Cacioppo, J.T. (2001). Can people feel happy and sad at the same time? Journal of Personality and Social Psychology, 81(4), 684-696. doi: 10.1037/00 22-3514.81.4.684.

Lewinsohn, P.M. \& Amenson, C.S. (1978). Some relations betwen pleasant and unpleasant mood-related events and depression. Journal of Abnormal Psychology, 87(6), 644-654. doi: 10.1037/0021-843X.87.6.644.

Lewinsohn, P.M. \& Graf, M. (1973). Pleasant activities and depression. Journal of Consulting and Clinical Psychology, 41(2), 261-268. doi: 10.1037/h0035142.

Meyer, T.D. \& Baur, M. (2009). Positive and negative affect in individuals at high and low risk for bipolar disorders. Journal of Individual Differences, 30(3), 169-175. doi: 10.1027/ 1614-0001.30.3.169.

Millon, T. \& Davis, R.D. (1998). Trastornos de la personalidad más allá del DSM-IV [Personality disorders beyond DSM-IV]. Barcelona: Masson.

Nolen-Hoeksema, S. (2000). The role of rumination in depressive disorders and mixed anxiety/depressive symptoms. Journal of Abnormal Psychology, 3, 504-511. doi:10.1037/00 21 843X.109.3.504.

Reich, J.W., Zautra, A.J. \& Davis, M. (2003). Dimensions of affect relationships: Models 
and their integrative implications. Review of General Psychology, 7(1), 66-83. doi: 10.10 37/1089-2680.7.1.66.

Rosenberg, E.L. (1998). Levels of analysis and the organization of affect. Review of General Psychology, 2(3), 247-270. doi:10.1037/10 89-2680.2.3.247.

Russell, J.A. (1980). A circumplex model of affect. Journal of Personality and Social Psychology, 39(6), 1161-1178. doi:10.1037/h0 077714.

Russell, J.A. \& Carroll, J.M. (1999). On the bipolarity of positive and negative affect. Psychological Bulletin, 125(1), 3-30. doi: 10.10 37/0033-2909.125.1.3

Sandín, B., Chorot, P., Lostao, L., Joiner, T.E., Santed, M.A. \& Valiente, R.M. (1999). Escalas PANAS de Afecto Positivo y Negativo: Validación factorial y convergencia transcultural [PANAS scales of positive and negative affect: Cross-cultural validation and convergence factor]. Psicothema, 11(1), 37-51.

Seligman, M.P.E. (1975). Helplessness: On depression, development, and death. San Francisco: W.H. Freeman and Co.

Spindler, H., Denollet, J., Kruse, C. \& Pedersen, S.S. (2009). Positive affect and negative affect correlate differently with distress and health-related quality of life in patients with cardiac conditions: Validation of the Danish Global Mood Scale. Journal of Psychosomatic Research, 67(1), 57-65. doi: 10.10 16/j.jp sychores.2008.11.003.

Strong, D.R., Kahler, C.W., Leventhal, A.M., Abrantes, A.M., Lloyd-Richardson, E.L., Niaura, R. \& Brown, R.A. (2009). Impact of bupropion and cognitive-behavioral treatment for depression on positive affect, negative affect, and urges to smoke during cessation treatment. Nicotine \& Tobacco Research, 11(10), 1142-1153. doi: 10.1093/ntr/ ntp111.

Tuccitto, D.E., Giacobbi, P.R. \& Leite, W.L. (2010). The internal structure of positive and negative affect: A confirmatory factor analysis of the PANAS. Educational and Psychological Measurement, 70(1), 125-141. doi: 10. 1177/0013164409344522.

Vallejo, J. (2006). Introducción a la Psicopatología y la Psiquiatría [Introduction to Psychopathology and Psychiatry] ( $6^{\mathrm{a}}$ ed). Barcelona: Editorial Masson \& Elsevier.

Van Etten, M.L., Higgins, S.T., Budney, A.J. \& Badger, G.J. (1998). Comparison of the frequency and enjoy ability of pleasant events in cocaine abusers vs. non-abusers using a standardized behavioral inventory. Addiction, 93 (11), 1669-1680. doi: 10.1046/j.1360-0443. 1998.931116695.x.

Versteeg, H., Pedersen, S.S., Erdman, R.A.M., van Nierop, J.W.I., de Jaegere, P. \& van Domburg, R.T. (2009). Negative and positive affect are independently associated with patient-reported health status following percutaneous coronary intervention. Quality of Life Research: An International Journal of Quality of Life Aspects of Treatment, Care \& Rehabilitation, 18(8), 953-960. doi: 10.1007/s111 36-009-9511-1.

Voogt, E., van der Heide, A., van Leeuwen, A.F., Visser, A.P., Cleiren, M.P.H.D., Passchier, J. $\&$ van der Maas, P.J. (2005). Positive and negative affect after diagnosis of advanced cancer. Psycho-Oncology, 14(4), 262-273. doi: 10.1002/pon.842.

Warr, P., Barter, J. \& Brownbridge, G. (1983). On the independence of positive and negative affect. Journal of Personality and Social Psychology, 44, 644-651. doi: 10.1037/0022-35 14.44.3.644.

Watson, D., Clark, L.A. \& Carey, G. (1988). Positive and negative affectivity and their relation to anxiety and depressive disorders. Journal of Abnormal Psychology, 97(3), 346353. doi: 10.1037/0021-843X.97.3.346.

Watson, D., Clark, L.A. \& Tellegen, A. (1988). Development and validation of brief measures of positive and negative affect: The PANAS 
Scales. Journal of Personality and Social Psychology, 54(6), 1063-1070. doi: 10.1037/ 0022-3514.54.6.1063.

Watson, D., Clark, L.A., Weber, K., Assenheimer, J.S., Strauss, M.E. \& McCormick, R.A. (1995). Testing a tripartite model: II. Exploring the symptom structure of anxiety and depression in student, adult, and patients samples. Journal of Abnormal Psychology, 104, 15-25. doi: 10.1037/0021-843X.104.1.15.

Wichers, M., Jacobs, N., Derom, C., Thiery, E. \& van Os, J. (2008). Depression: Too much negative affect or too little positive affect? Twin Research and Human Genetics, 10(Suppl), 19-20. doi: 10.1375/twin.10.supp.19.

Windsor, T.D. \& Anstey, K.J. (2010). Age differences in psychosocial predictors of positive and negative affect: A longitudinal investigation of young, midlife, and older adults. Psychology and Aging, 25(3), 641-652. doi: 10. 1037/a0019431.
Zautra, A.J., Burleson, M.H., Smith, C.A., Blalock, S.J., Wallston, K.F., DeVellis, R.F., DeVellis, B.M. \& Smith, T.W. (1995). Arthritis and perceptions of quality of life: An examination of positive and negative affect in rheumatoid arthritis patients. Health Psychology, 14, 399-408. doi: 10.1037/02786133.14. 5.399 .

Zautra, A.J., Potter, P.T. \& Reich, J.W. (1997). The independence of affects is contextdependent: An integrative model of the relationship between positive and negative affect. En M.P. Lawton, K.W. Schaie \& M.P. Lawton (Eds.), Annual Review of Gerontology and Geriatrics: Vol. 17. Focus on Adult Development (pp. 75-103). New York: Springer. Zautra, A.J., Reich, J.W., Davis, M.C., Nicolson, N.A. \& Potter, P.T. (2000). The role of stressful events in the relationship between positive and negative affect. Evidence from field and experimental studies. Journal of Personality, 68, 927-951. doi: 10.1111/1467-6494.00121. 